\title{
Estresse, competência e problemas psicológicos de adolescentes estudantes
}

\author{
Stress, competence and psychological problems in adolescent students
}

Nancy Ramacciotti de Oliveira-Monteiro', Maria Aznar-Farias', Camila de Almeida Nava', Juliana Olivetti Guimarães Nascimento ${ }^{1,2}$, Fábio Tadeu Montesano', Regina Célia Spadari-Bratfisch ${ }^{3}$

\begin{abstract}
Resumo
Introdução: 0 desenvolvimento positivo resulta em competências em diferentes domínios (cognitivos, afetivos, sociais) importantes para a proteção frente a problemas psicológicos e de comportamento que podem levar a disfunções, prejudicando a organização de ações, percepções, atitudes e interação com o meio. Objetivo: Avaliar de forma descritiva indicadores de estresse, competência social e problemas psicológicos e comportamentais em um grupo de adolescentes estudantes em jornada integral. Método: Este estudo incluiu 50 adolescentes (22 meninos e 28 meninas com idades de 12 a 18 anos). 0 s instrumentos usados foram o ASQ (Questionário de estresse para adolescentes) e o YSR (Inventário de autoavaliação para adolescentes). Dados relativos à competência social e a problemas psicológicos e de comportamento foram sistematizados com uso do software do YSR, enquanto a organização dos resultados do ASO seguiu padrões descritivos de frequência dos dados da escala tipo Likert do instrumento. 0 estudo da associação entre ASQ e as variáveis do YSR foi feito a partir da observação de gráficos de dispersão e do cálculo do coeficiente de correlação linear de Pearson. Resultados: Verificou-se tendência geral à baixa intensidade de estresse e à faixa não clínica para problemas psicológicos e para competência total (que inclui competência social). No subgrupo dos meninos foi verificada correlação moderada entre problemas psicológicos e estresse. Conclusão: Embora tenham sido detectadas queixas relacionadas ao tempo de ficar na escola, a inserção escolar em período integral pode ter sido importante variável interveniente para os resultados positivos encontrados, o que aponta necessidades de ampliação de estudos na temática.
\end{abstract}

Palavras-chave: desenvolvimento positivo; adolescência; competência; estresse; psicologia positiva.

\begin{abstract}
Introduction: Positive development results in acquiring competence in different domains (cognitive, affective, social), that are important protection factors to psychological and behavioral problems, which might lead to anomalies in development, damaging the organization of actions, perceptions, attitudes and the interactions with the environment. Objective: To evaluate comprised perceptions related to stress, social competence, psychological and behavioral problems in a group of adolescent students in integral shift. Method: This study included 50 adolescents (22 boys, 28 girls, aged 12-18 years). The instruments used were ASQ (Adolescent stress questionnaire) and YSR (Youth self report). Data related to social competence and psychological problems were systematized with the use of the software YSR, while the organization of the results obtained from ASQ followed the standards described for the frequency of data from the Likert scale from the instrument. The study of the association between ASQ and the variables from YSR was made from the observation of dispersion graphics and the calculation of the Pearson linear correlation coefficient. Results: A general trend was verified to low intensity of stress to the non clinical level for psychological problems and to total competence (including social competence). A moderate correlation between psychological problems and stress was identified within the boys subgroup. Conclusion: Although some complaints about the excessive time in school were related, the permanence in school for an integral shift might have been an important variable intervening for the positive results found, which points to the necessity to increase the studies in this theme.
\end{abstract}

Keywords: positive development; adolescence; competence; stress; positive Psychology.

Recebido em: 28/01/2012

Revisado em: 19/02/2012

Aprovado em: 22/02/2012

Trabalho realizado na Universidade Federal de São Paulo (UNIFESP) - Santos (SP), Brasil. Pesquisa parcialmente apresentada no $9^{\circ}$ Congresso Nacional de Psicologia da Saúde, Portugal, fevereiro de 2012. Resumo expandido publicado nas Actas do Congresso.

'Laboratório de Psicologia Ambiental e Desenvolvimento Humano da Universidade Federal de São Paulo (UNIFESP) - Santos (SP), Brasil.

2Programa de Pós-Graduação em Psiquiatria e Psicologia Médica da UNIFESP - São Paulo (SP), Brasil.

${ }^{3}$ Laboratório da Biologia do Estresse da UNIFESP - Santos (SP), Brasil.

Endereço para correspondência: Nancy Ramacciotti de Oliveira-Monteiro - Avenida Ana Costa, 95 - Vila Mathias - CEP: 11060-001 - Santos (SP),

E-mail: nancy.unifesp@gmail.com

Fonte de financiamento: Universidade Federal de São Paulo (UNIFESP)/2009, com processo vinculado ao Programa Institucional de Bolsa de Iniciação Científica (PIBIC/CNPq).

Conflito de interesse: nada a declarar. 


\section{Introdução}

A literatura sobre desenvolvimento humano vem, há muito tempo, descrevendo a adolescência como a etapa da vida marcada caracteristicamente por conflitos internos e interpessoais. Desde o clássico trabalho de Stanlley Hall, os problemas desse estágio vital têm sido considerados pela Psicologia como próprios e sugestivos da transição da infância para a vida adulta. Nessa linha de pensamento, encontramse contribuições como a de Anna Freud ${ }^{1}$, que na década de 1930 destacou os estados de ansiedade e os mecanismos defensivos comuns da adolescência, e de Aberastury et al. ${ }^{2}$, também autores de orientação psicanalítica, que propuseram uma chamada "síndrome da adolescência normal”, mostrando que aspectos problemáticos e instabilidades são transtornos comuns da segunda década do ciclo vital.

O conceito de crise de identidade da adolescência, parte da teoria psicossocial de Erikson ${ }^{3}$ sobre as idades da vida, também contribuiu para a visão caracteristicamente conflitiva da etapa vital da adolescência, talvez por uma saturação semântica de aspectos negativos da palavra "crise". Para o estudioso, a adolescência é idade marcada por uma crise de identidade, num período em que, ao lado da exploração de si e do mundo, ocorre o chamado estado de "moratória", que permite postergação das reponsabilidades que serão assumidas quando adulto. Entretanto, crise, para Erikson, tem o sentido de uma encruzilhada em determinados pontos do desenvolvimento nas etapas da vida, possuindo vertentes positivas ao lado das negativas. O polo negativo da crise da adolescência é referente a uma possível não superação da difusão de identidade (parte inicial do desenvolvimento da identidade), falha que levaria a dificuldades para o indivíduo aprender sobre si mesmo e seu entorno e para construir uma identidade realista em consonância com suas características pessoais e seu contexto sociocultural. Por outro lado, para o autor, a crise da adolescência tem seu polo positivo, enquanto vai permitir a escolha e desenvolvimento de valores para a vida, o que fará os jovens perceberem que suas realizações possuem reconhecimento e significado em sua cultura ${ }^{4}$.

Desde o final do século passado, outra vertente de trabalhos de psicologia evolutiva vem questionando a visão problemática, e de certa forma negativa (em alguns casos, até psicopatológica), da adolescência, como, por exemplo, a contribuição de Hoffman et al. ${ }^{5}$ que relativiza mitos concernentes à adolescência, presentes desde Aristóteles e Shakespeare. A potência positiva de desenvolvimento presente na adolescência é citada por muitos autores ${ }^{6,7,8}$, incluindo estudiosos do desenvolvimento de adolescentes em condições de vulnerabilidade ${ }^{9}$. Esses trabalhos privilegiam o enfoque da Psicologia positiva, cujo advento trouxe uma alteração do paradigma negativo (o que destaca déficits e problemas) para um novo paradigma, com destaque para o lado positivo do desenvolvimento humano (os recursos) e dos ativos que promovem esse desenvolvimento positivo ${ }^{6}$. Pela perspectiva da Psicologia positiva, problemas e recursos humanos não são dimensões opostas e devem ser considerados como provenientes de sistemas psicológicos distintos ${ }^{10}$.
O desenvolvimento global da pessoa envolve o estudo da competência, entendida como a capacidade para mostrar desempenho em diferentes áreas de atividade (social, acadêmica, cognitiva e vocacional). Habilidades sociais, como a assertividade, facilidade para resolução de conflitos e capacidade para tomar decisões, fazem parte do conjunto de competências próprias do desenvolvimento humano ${ }^{6}$. A competência social, uma das dimensões da competência, é definida como um conjunto de habilidades sociocognitivas e de conhecimento (incluindo a capacidade de controle emocional) que fazem mediação de comportamentos específicos, julgados como efetivos e idôneos, por outras pessoas e pelo próprio sujeito $^{11}$. A competência social engloba processos complexos, habilidades e estratégias de diferentes dimensões. Ela contribui para um adequado ajuste social ${ }^{12}$ e é tida como componente chave da personalidade saudável e feliz ${ }^{11}$. A competência social é um elemento da dimensão positiva do desenvolvimento humano, produto resultante desse desenvolvimento e fator de proteção diante de problemas psicológicos e de comportamento, aqueles que prejudicam a organização de ações, percepções, atitude e interação com o meio ${ }^{13}$.

Os transtornos e problemas psicológicos e comportamentais emergem como expressões de disfunções no desenvolvimento em todas as etapas do ciclo vital. Estes transtornos podem ser classificados como de ordem internalizante (manifestados por perturbações emocionais e cognitivas), de ordem externalizante (problemas comportamentais ou de atuação, como nos comportamentos antissociais) e de uso/abuso de determinadas substâncias (drogas) ${ }^{14}$.

O estresse é, por vezes, classificado como problema psicológico enquanto dimensão paralisante, consequência de eventos negativos muito constantes ou de maior carga de intensidade, com capacidade para prejudicar estados de saúde. Nesse sentido, o estresse é entendido como um mal-estar causado por fatores físicos e emocionais, gerando nervosismo, tensão, cansaço e esgotamento. No entanto, também tem uma dimensão positiva, enquanto elemento presente nos desafios que propulsionam aquisição de habilidades e superação de obstáculos e adversidades. Os significados do estresse possuem enfoques orgânicos, quando se refere a aspectos fisiológicos e bioquímicos associados, e/ou psicossociais, mais voltados para os fatores externos, causadores do estresse, os eventos estressores. O impacto dos eventos estressores é determinado pela forma como ocorrem e também como são percebidos. No tocante aos eventos estressores, devem ser considerados, ainda, os fatores mediadores, como as características individuais e o suporte afetivo e social percebido nos contextos de desenvolvimento ${ }^{15}$.

A ativação do sistema de estresse pode funcionar como agente protetor enquanto prepara o organismo para atacar ou fugir, aumentando as chances de sobrevivência. Porém, quando um determinado limiar é ultrapassado, este mesmo sistema pode mudar de ação protetora para danosa, podendo desencadear problemas. Normalmente a resposta aguda de estresse é limitada e de curta duração, tem efeitos temporários e não acarreta problemas à integridade do indivíduo. Entretanto, a resposta crônica de estresse, caracterizada por ativação constante do sistema de estresse, tem 
como resultado a secreção aumentada ou prolongada do hormônio liberador de corticotrofina (CRH), hormônio adrenocorticotrófico (ACTH) e de glicocorticóides, podendo acarretar prejuízos ao organismo ${ }^{16}$.

Uma resposta apropriada do sistema de estresse é condição indispensável para proporcionar sensação de bem-estar, desempenho apropriado às tarefas e interação social positiva. Inversamente, respostas inapropriadas do sistema de estresse podem causar prejuízo ao crescimento e ao desenvolvimento do indivíduo e acarretar uma variedade de disfunções endócrinas, metabólicas, imunológicas e comportamentais. O desenvolvimento e a gravidade destas disfunções dependem da vulnerabilidade genética do indivíduo, da sua exposição adicional a fatores ambientais adversos e da ocorrência concomitante de eventos estressores ${ }^{17}$.

Os eventos estressores que ocorrem com maior frequência em crianças e adolescentes estão relacionados aos contextos escolar e familiar $^{15}$, que, de acordo com a teoria ecológica do desenvolvimento ${ }^{18}$, constituem-se como os principais microssistemas nos quais são estabelecidas as relações face-a-face e de reciprocidade. Entre os estressores que estariam relacionados ao período da adolescência, Kristensen et al. ${ }^{19}$ também apontam os conteúdos relativos à família e à escola, além daqueles relacionados a mudanças corporais típicas da puberdade, doença ou morte de pessoas próximas.

À luz dessas considerações, apresenta-se um estudo que teve por objetivo avaliar de forma descritiva um grupo de adolescentes no tocante aos indicadores de estresse, competência social e problemas psicológicos e comportamentais.

\section{Método}

\section{Amostra}

Foram investigados 50 adolescentes com idade entre 12 e 18 anos, sendo 28 do sexo feminino e 22 do masculino, que estudavam em regime de tempo integral (manhã/tarde) em classes de escola pública de Ensino Fundamental e Médio de uma cidade da Baixada Santista (SP). A caracterização socioeconômica da amostra foi avaliada pelo Critério Brasil ${ }^{20}$, apresentando indicadores de maior incidência nas classes C (36\%) e B2 (32\%).

\section{Instrumentos}

Para exame do perfil de estresse, competências sociais e problemas psicológicos e comportamentais foram utilizados dois instrumentos: o ASQ (Questionário de estresse percebido para adolescentes) e o YSR (Inventário de autoavaliação para adolescentes), abaixo descritos.

1. ASQ - o "Questionário de estresse percebido para adolescentes" tem por objetivo traçar o perfil de estresse de adolescentes em idade escolar $^{21}$. Foi desenvolvido por meio de construção de instrumentos prévios de informações que se refletem como fatores estressores para adolescentes estudantes e apresenta 62 afirmações diretas para escolhas em uma escala de tipo Likert (numerada de um a cinco) re- ferente a graus de intensidade (mais baixos a mais altos) do estresse percebido com relação a situações e sintomas indicados na afirmação. $\mathrm{O}$ instrumento tem 11 escalas que avaliam estresse relativo à vida familiar, performance escolar, frequência à escola, relações românticas, pressão dos pares, interação com professores, incerteza do futuro, conflito escola/lazer, pressão financeira, responsabilidades emergentes da vida adulta e relações sociais. O trabalho de validação brasileira do instrumento vem incluindo correlações de âmbito biológico, com análises laboratoriais da concentração salivar de cortisol $^{16}$. Com concordância e permissão dos autores para adaptação brasileira, além de tradução e validação semântica, foram incluídos itens à escala original de origem australiana ${ }^{22}$.

2. YSR - o "Inventário de autoavaliação para adolescentes de 11 a 18 anos”, instrumento da Bateria do Sistema da Avaliação Empiricamente Baseada de Achenbach (ASEBA) ${ }^{23,24}$, com questões fechadas e abertas, avalia o funcionamento psicossocial do adolescente em escalas relativas a competência e a problemas emocionais e comportamentais (distúrbios internalizantes e externalizantes). $\mathrm{Na}$ área de competência social global, o questionário investiga participação em atividades (esportes, passatempos) e em grupos, e relacionamento familiar e de amizades. A área de problemas emocionais e comportamentais inclui avaliação de ansiedade, depressão, retraimento, queixas somáticas, problemas com o contato social, com o pensamento e com a atenção, além de violação de regras e comportamento agressivo. O instrumento oferece também uma avaliação de problemas psicológicos e comportamentais em escalas orientadas pelo DSM-IV (Diagnostic and Statistical Manual of Mental Disorders), incluindo identificação de perturbações de sono, de linguagem e alimentação. O YSR foi validado para a população brasileira por Rocha ${ }^{25}$.

\section{Procedimentos}

O estudo foi aprovado pelo Comitê de Ética em Pesquisa da Universidade Federal de São Paulo (CEP/UNIFESP - no 1209/09). Os pais/ responsáveis dos adolescentes que aceitaram participar assinaram termos de consentimento livre e esclarecido para a inclusão dos adolescentes no estudo. A aplicação dos instrumentos ocorreu de forma coletiva, em sala de aula. Após a ação, que durou aproximadamente 40 minutos, uma das pesquisadoras do estudo, ainda em sala de aula, verificou se os instrumentos tinham sido completamente respondidos, prestando mais esclarecimentos e solicitando que as respostas fossem completadas, quando necessário.

O procedimento de tratamento dos dados do ASQ incluiu análises dos resultados derivados da soma das respostas de todos os itens das escalas e distribuição delas por categorias de intensidade de estresse: baixa (graus um e dois da Escala Likert), média (grau três) e alta (graus quatro e cinco). Para correção do inventário YSR foi utilizado o software específico da Bateria ASEBA (Assessment Data Manager Program - ADM), que permite sistematização dos dados em perfis nas categorias: não clínica, clínica e limítrofe. 
Para analisar os resultados de meninas e meninos em diferentes faixas etárias com relação a cada uma das variáveis componentes do ASQ e do YSR, empregou-se o modelo de análise de variância com dois fatores fixos. O estudo da associação entre ASQ e as variáveis do YSR foi realizado a partir da observação de gráficos de dispersão e do cálculo do coeficiente de correlação linear de Pearson.

\section{Resultados}

Os resultados do ASQ indicaram faixas de estresse de intensidade baixa a moderada, nas 11 escalas do instrumento. O subgrupo das meninas investigadas manteve a distribuição de dados do ASQ concentrada no grau baixo de intensidade em todas as suas escalas. No subgrupo dos meninos, a distribuição dos dados sobre estresse do conflito escola/lazer apresentou escores praticamente equivalentes para os graus de intensidade baixa e alta. Da mesma forma ocorreu com relação ao estresse da pressão financeira, para o qual a distribuição dos resultados dos meninos também esteve nas intensidades baixa e alta. Meninos e meninas diferiram quanto à variável, com dados de intensidade de estresse mais presentes nos meninos.

A Tabela 1 apresenta resultados obtidos nas escalas do ASQ e a comparação deles nos meninos e meninas da amostra.

Com relação ao YSR, o grupo investigado demonstrou resultados com tendência à faixa não clínica nas duas escalas do instrumento (a de Competência e a de Problemas psicológicos/de comportamento). $\mathrm{Na}$ parte qualitativa, de forma geral, os adolescentes da amostra referiram que se relacionavam positivamente com outros adolescentes (dentro e fora do contexto escolar), tinham atividades esportivas e passatempos. A maioria deles desempenhava alguma atividade em casa (ajuda nos afazeres doméstico) e poucos relataram exercer atividades remuneradas. Questionados acerca de preocupações ou problemas com a escola, de forma recorrente, apontaram apreensão com fracasso escolar e reprovações, além de referirem queixas relacionadas ao tempo de ficar na escola (manhã e tarde). Problemas de relacionamento entre os alunos, violência, falta de motivação e insatisfação com a escola e professores também foram apresentados em respostas a questões abertas do YSR.

Na escala de Competência do YSR, que abrange amizade, responsabilidades, participação em grupos, tarefas realizadas habitualmente, esportes e passatempos, a distribuição dos dados da amostra (meninos e meninas) indicou tendência à faixa não clínica. No tocante às Atividades, uma parcela dos meninos apresentou escores limítrofes. A distribuição dos dados sobre Competência total, que engloba competências sociais e atividades, mostrou que a grande maioria dos meninos encontrava-se na faixa não clínica e limítrofe enquanto uma pequena parcela atingiu escores clínicos. Das adolescentes meninas, apenas um pequeno segmento atingiu a faixa limítrofe, com concentração da distribuição dos resultados na faixa não clínica.

A Tabela 2 apresenta medidas descritivas de escalas do YSR concernentes à Competência e a Problemas psicológicos/comportamentais, incluindo os internalizantes e externalizantes em meninos e meninas da amostra estudada. As médias gerais obtidas situam-se na faixa não clínica (escores acima de 40) dos resultados relativos às escalas de Competência e também na faixa não clínica (escores abaixo de 64) nas escalas relativas a problemas.

Tabela 1: Médias dos escores nas escalas do Questionário de estresse para adolescentes (ASQ): comparação entre meninos e meninas

\begin{tabular}{|c|c|c|c|c|c|c|c|}
\hline \multirow{3}{*}{$\begin{array}{l}\text { Variável } \\
\text { Vida familiar }\end{array}$} & \multirow{3}{*}{$\begin{array}{c}\text { Média } \\
\text { DP }\end{array}$} & \multirow{3}{*}{$\begin{array}{c}\text { Meninos } \\
28,8 \\
9,5\end{array}$} & \multirow{3}{*}{$\begin{array}{c}\text { Meninas } \\
30,1 \\
12,7\end{array}$} & \multirow{3}{*}{$\begin{array}{c}p \\
0,957\end{array}$} & \multirow{3}{*}{$\begin{array}{c}\text { Diferença } \\
-0,2\end{array}$} & \multicolumn{2}{|c|}{ Intervalo de confiança } \\
\hline & & & & & & 65 & 61 \\
\hline & & & & & & $-0,5$ & 0,1 \\
\hline \multirow{2}{*}{ Performance escolar } & Média & 18,4 & 16,7 & \multirow{2}{*}{0,508} & \multirow{2}{*}{1,3} & \multirow{2}{*}{$-2,6$} & \multirow{2}{*}{5,2} \\
\hline & $\mathrm{DP}$ & 7,2 & 6,5 & & & & \\
\hline \multirow{2}{*}{ Frequentar a escola } & Média & 9,0 & 7,9 & \multirow{2}{*}{0,224} & \multirow{2}{*}{1,3} & \multirow{2}{*}{$-0,8$} & \multirow{2}{*}{3,5} \\
\hline & $\mathrm{DP}$ & 4,3 & 3,0 & & & & \\
\hline \multirow{2}{*}{ Relações românticas } & Média & 11,6 & 10,7 & \multirow{2}{*}{0,204} & \multirow{2}{*}{1,9} & \multirow{2}{*}{$-1,1$} & \multirow{2}{*}{4,9} \\
\hline & $\mathrm{DP}$ & 5,3 & 5,1 & & & & \\
\hline \multirow{2}{*}{ Pressão dos pares } & Média & 16,0 & 15,4 & \multirow{2}{*}{0,745} & \multirow{2}{*}{0,7} & \multirow{2}{*}{$-3,4$} & \multirow{2}{*}{4,7} \\
\hline & $\mathrm{DP}$ & 6,6 & 7,5 & & & & \\
\hline \multirow{2}{*}{ Interação com professores } & Média & 15,8 & 15,5 & \multirow{2}{*}{0,730} & \multirow{2}{*}{0,6} & \multirow{2}{*}{$-3,1$} & \multirow{2}{*}{4,4} \\
\hline & $\mathrm{DP}$ & 6,4 & 6,7 & & & & \\
\hline \multirow{2}{*}{ Incerteza do futuro } & Média & 7,5 & 6,4 & 0491 & 06 & -12 & 24 \\
\hline & DP & 3,4 & 2,8 & 0,491 & 0,0 & $-1,2$ & 2,4 \\
\hline & Média & 13,9 & 12,5 & 0107 & & & \\
\hline Conflito escola/lazer & $\mathrm{DP}$ & 6,1 & 5,8 & 0,192 & 2,2 & $-1,1$ & 5,6 \\
\hline Preccãn financoira & Média & 10,4 & 7,8 & 033 & 30 & 03 & 58 \\
\hline 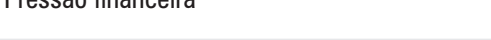 & DP & 5,6 & 4,0 & 0,000 & 0,0 & , & 0,0 \\
\hline Resnoncabilidades emorrentes da vida adulta & Média & 6,8 & 6,3 & 0679 & 04 & -14 & 21 \\
\hline Kesponsabilidades emergentes da vida aduita & $\mathrm{DP}$ & 3,0 & 2,9 & $0,6 / 9$ & 0,4 & $-1,4$ & 2,1 \\
\hline Relacõec sociaic & Média & 8,4 & 8,1 & 0630 & & & \\
\hline Relaçoes socıaıs & $\mathrm{DP}$ & 4,8 & 4,3 & 0,639 & 0,6 & $-2,0$ & 3,2 \\
\hline Tntal $-A S n$ & Média & 146,5 & 137,4 & 0359 & 125 & 146 & 395 \\
\hline 管 & DP & 46,4 & 49,7 & 0,0039 & $12, J$ & $-14,0$ & $39, \mathrm{~J}$ \\
\hline
\end{tabular}


$\mathrm{Na}$ área de Problemas psicológicos indicados pelo YSR, os dados da amostra distribuíram-se na faixa não clínica em sete dos oito agrupamentos avaliados pelo instrumento (ansiedade/depressão, retraimento/depressão, queixas somáticas, problemas com o contato social, problemas com o pensamento, problemas com a atenção, violação de regras e comportamento agressivo). Apenas na área de problemas sociais houve indicador de faixa limítrofe no subgrupo dos meninos.

Quando analisados os problemas internalizantes e externalizantes da amostra, os dados encontrados nos protocolos dos meninos apresentaram escores clínicos referentes aos problemas internalizantes. No subgrupo das meninas, tanto em relação aos problemas internalizantes quanto aos externalizantes, os dados estiveram distribuídos nas faixas não clínica e limítrofe.

Uma distribuição da amostra na faixa não clínica também foi encontrada em relação aos oito agrupamentos de Problemas psicológicos e de Comportamento, em perfis orientados pelo DSM-IV do YSR, que incluem transtornos afetivos, transtornos de ansiedade, problemas somáticos, déficit de atenção/transtorno de hiperatividade, transtorno opositor desafiante, problemas de conduta, problemas obsessivos compulsivos e problemas de estresse pós-traumático. Dos oito agrupamen-

Tabela 2: Medidas descritivas de resultados do Inventário de autoavaliação para adolescentes (YSR) em meninos e meninas

\begin{tabular}{|c|c|c|c|c|c|c|c|}
\hline \multirow{3}{*}{$\begin{array}{l}\text { Variável } \\
\text { Atividades }\end{array}$} & \multirow{3}{*}{$\begin{array}{c}\text { Média } \\
\text { DP }\end{array}$} & \multirow{3}{*}{$\begin{array}{l}\text { Meninos } \\
42,1 \\
11,6\end{array}$} & \multirow{3}{*}{$\begin{array}{l}\text { Meninas } \\
48,7 \\
10,5\end{array}$} & \multirow{3}{*}{$\begin{array}{c}p \\
0,100\end{array}$} & \multirow{3}{*}{$\begin{array}{l}\text { Diferença } \\
-5,5\end{array}$} & \multicolumn{2}{|c|}{ Intervalo de confiança } \\
\hline & & & & & & & \\
\hline & & & & & & $-12,0$ & 1,1 \\
\hline \multirow{2}{*}{ Social } & Média & 45,5 & 50,3 & \multirow{2}{*}{0,174} & \multirow{2}{*}{$-3,9$} & \multirow[b]{2}{*}{$-9,6$} & \multirow{2}{*}{1,8} \\
\hline & DP & 10,1 & 9,4 & & & & \\
\hline \multirow{2}{*}{ Acadêmico } & Média & 2,0 & 2,1 & \multirow{2}{*}{0,855} & \multirow{2}{*}{$-0,1$} & \multirow{2}{*}{$-0,3$} & \multirow{2}{*}{0,3} \\
\hline & DP & 0,4 & 0,6 & & & & \\
\hline \multirow{2}{*}{ Competência Total } & Média & 41,3 & 49,0 & \multirow{2}{*}{0,071} & \multirow{2}{*}{$-6,5$} & \multirow{2}{*}{$-13,5$} & 06 \\
\hline & DP & 11,5 & 11,9 & & & & 0,0 \\
\hline & Média & 61,1 & 58,9 & & & & \\
\hline Ansiedade/depressao & DP & 8,4 & 8,5 & 0,242 & 2,9 & $-2,0$ & 7,7 \\
\hline Jcolamento/denreccão & Média & 59,1 & 56,4 & 0107 & 27 & 00 & 81 \\
\hline Isolamento/aepressao & DP & 8,4 & 7,0 & 0,102 & 3,1 & $-0,8$ & 8,1 \\
\hline Oueixas somáticas & Média & 57,7 & 57,0 & 0502 & 19 & -36 & 73 \\
\hline unelxas somalicas & DP & 10,2 & 8,6 & 0,002 & 1,9 & $-3,0$ & 1,5 \\
\hline & Média & 58,7 & 56,4 & & & & \\
\hline Problemas socials & DP & 7,4 & 7,1 & 0,103 & 3,4 & $-0, I$ & 1,5 \\
\hline Problomas do noncomonto & Média & 57,5 & 55,0 & 0166 & 27 & 12 & 67 \\
\hline Problemas de pensamento & DP & 7,7 & 5,4 & 0,166 & $2, I$ & $-1,2$ & 6,1 \\
\hline Problemas de atencãn & Média & 56,3 & 56,4 & 0565 & 11 & -28 & 50 \\
\hline Probiemas de atençao & DP & 6,5 & 7,2 & 0,565 & & & 3,0 \\
\hline Comnortamento de guehra de reara & Média & 54,6 & 54,1 & $0, \Delta 40$ & 13 & 46 & 20 \\
\hline Comportamento de quebra de regra & DP & 6,0 & 5,4 & 0,440 & $-1,3$ & $-4,6$ & 2,0 \\
\hline Comnortamento a aroccivo & Média & 56,4 & 57,4 & $0: 81$ & 17 & 66 & $31+2>>0>0$ \\
\hline Comportamento agressivo & DP & 7,1 & 9,9 & 0,481 & $-1, I$ & $-6,6$ & 3,1 \\
\hline & Média & 59,1 & 56,4 & & & & \\
\hline Problemas internalizantes & DP & 11,1 & 10,9 & 0,188 & 4,2 & $-2,1$ & 10,5 \\
\hline Problemas externalizantec & Média & 52,7 & 53,4 & $0 ? 20$ & 20 & 00 & 21 \\
\hline Problemas externalizantes & DP & 10,4 & 10,6 & 0,342 & $-2,9$ & $-8,8$ & 3,1 \\
\hline Total de nroblomac & Média & 56,4 & 54,6 & 05010 & 10 & 15 & 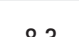 \\
\hline lotal de problemas & DP & 11,3 & 10,8 & 0,548 & 1,9 & $-4,5$ & 8,3 \\
\hline Tranctornes ofotives & Média & 58,1 & 56,1 & 0100 & 31 & 12 & 70 \\
\hline Iranstornos afetivos & DP & 8,6 & 7,1 & 0,144 & 3,4 & $-1,2$ & 7,9 \\
\hline Tranctornec do anciodado & Média & 59,0 & 58,6 & & & & \\
\hline Iranstornos de ansiedade & DP & 7,4 & 7,5 & 0,556 & 1,3 & $-3,1$ & 5,6 \\
\hline Problemas comáticncs & Média & 57,0 & 56,0 & 0023 & 12 & 20 & 65 \\
\hline Frodiemas somaticos & DP & 9,4 & 8,3 & $0,0<3$ & 1,3 & $-3,9$ & 0,5 \\
\hline Dóficit do atoncว̃o/hinoratividado & Média & 55,7 & 55,5 & 0710 & & & $A^{3}+2>>0$ \\
\hline Defıcıt de atençao/hıperatıvıdade & DP & 6,9 & 5,3 & $0, / 10$ & 0,1 & $-2,9$ & 4,3 \\
\hline Tranctorno onecitor decafiante & Média & 54,6 & 55,5 & 0.500 & 12 & 52 & 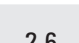 \\
\hline Iranstomo opositor desalante & DP & 5,9 & 7,5 & 0,500 & $-1,3$ & $-5,2$ & 2,6 \\
\hline Problemas de conduta & Média & 55,6 & 55,2 & 0438 & -15 & -55 & 24 \\
\hline Frodientas ue contunta & DP & 7,2 & 6,4 & & & & \\
\hline Problemas obsessivo-compulsivos & Média & 59,8 & 56,5 & 0.123 & 30 & -0.8 & 68 \\
\hline & DP & 6,7 & 6,3 & $0,1<3$ & 3,0 & $-0,8$ & \\
\hline Problemas de estrecse nós-traumático & Média & 60,3 & 57,1 & 0122 & 3 & 11 & 00 \\
\hline rrodiemas de estresse pos-iraumatico & DP & 9,1 & 8,4 & $0,12 L$ & 3,9 & $-1,1$ & ,, 0 \\
\hline Oualidades positivas & Média & 51,0 & 52,8 & 0827 & 07 & 57 & 71 \\
\hline Uualiuaues pusitivas & DP & 12,2 & 10,2 & $0,0<1$ & 0,1 & $-3,1$ & 1,1 \\
\hline
\end{tabular}


tos avaliados, apenas a distribuição dos dados sobre transtornos de ansiedade e problemas de estresse pós-traumático indicou um segmento dos meninos na faixa limítrofe, enquanto a maioria da distribuição dos dados do total da amostra encontrava-se na faixa não clínica.

A análise da associação entre resultados do ASQ e do YSR indicou correlação moderada entre estresse (ASQ) e problemas internalizantes (YSR), e entre estresse (ASQ) e total de problemas (YSR), no subgrupo dos meninos. Não houve associação entre os resultados relativos a estresse (ASQ) e a competência (YSR). A Tabela 3 apresenta coeficientes de correlação linear calculados entre resultados do ASQ e do YSR em meninos e meninas da amostra.

\section{Discussão}

Os adolescentes estudantes investigados, em sua maioria, apresentaram tendências positivas de desenvolvimento, especialmente no tocante à avaliação da competência social e do nível de intensidade baixa de estresse. Isso vem ao encontro das contribuições colocadas por Hoffman et al..$^{5}$ quando afirmaram que existem mitos negativos sobre a adolescência que não descrevem a maioria dos adolescentes. Kimmel et al. ${ }^{6}$ também questionaram a visão, de certa forma dramática, da adolescência enquanto etapa evolutiva, apontando consequências indesejáveis. Uma delas situa-se em uma possível contradição entre esperar dos jovens maior participação nas tomadas de decisão que afetam aspectos familiares, escolares e sociais, e ter deles uma imagem de grupo social caracteristicamente conflitivo.

Meninos e meninas da amostra investigada neste estudo indicaram ter percepção positiva de suas qualidades (YSR). Um estudo de Assis et al. ${ }^{26}$ mostrou que adolescentes possuem uma visão positiva de si próprios, a despeito da visão que os adultos e a sociedade em geral têm deles. Minayo ${ }^{27}$ apontou que a imagem mais positiva que os jovens parecem ter de si mesmos pode funcionar como uma defesa perante a negatividade presente na visão que a sociedade adulta tem sobre a juventude.

Como já referido, Trianes et $\mathrm{al}^{11}$ destacam a competência social como elemento da personalidade saudável e feliz. Os dados relativos à competência social investigada na amostra indicaram de forma geral

Tabela 3: Coeficientes de correlação linear de Pearson entre Questionário de estresse para adolescentes (ASO) e as variáveis do Inventário de autoavaliação para adolescentes (YSR) segundo o sexo

\begin{tabular}{lcccc}
\hline Variável & Sexo & Coeficiente & \multicolumn{2}{c}{ Intervalo de confiança } \\
\hline \multirow{2}{*}{ Total de competências } & Masculino & 0,304 & $-0,113$ & 0,630 \\
& Feminino & 0,246 & $-0,208$ & 0,613 \\
\multirow{3}{*}{ Problemas internalizantes } & Masculino & 0,540 & 0,210 & 0,760 \\
& Feminino & 0,009 & $-0,414$ & 0,429 \\
\multirow{2}{*}{ Problemas externalizantes } & Masculino & 0,259 & $-0,126$ & 0,576 \\
\cline { 2 - 5 } Total de problemas & Feminino & 0,240 & $-0,202$ & 0,601 \\
& Masculino & 0,520 & 0,183 & 0,748 \\
& Feminino & 0,073 & $-0,359$ & 0,480 \\
\hline
\end{tabular}

graus positivos (não clínicos) concernentes a tal competência (YSR), o que apontou para uma positividade no desenvolvimento daqueles adolescentes. Esse indicativo pode ter tido associação com a condição da amostra, em termos de jornada de estudo em tempo integral. O tempo diário de permanência na escola em horário integral pode permitir e facilitar o desenvolvimento de comportamentos sociais competentes, pela oportunidade de criação e aprofundamento de laços no grupo de iguais, além da inserção em atividades educativas, sociais e esportivas. A jornada escolar integral traz importante reflexão sobre as funções que cabem à escola em seu papel na formação para a vida em sociedade ${ }^{28}$.

Diferente do encontrado por Poletto et al. ${ }^{15}$, que investigaram eventos estressores em crianças e adolescentes em situação de vulnerabilidade social, na amostra do presente estudo (adolescentes estudantes), os eventos relativos à família foram referidos de forma geral como pouco estressantes, tanto pelos meninos quano pelas meninas, da mesma forma que o foram eventos relativos a relações românticas, pressão dos pares, incerteza do futuro, responsabilidades emergentes da vida adulta e relações sociais (ASQ). Dentre as quatro escalas do ASQ relativas a eventos estressores na escola, os resultados de maior intensidade de estresse foram encontrados na escala relativa ao conflito escola/lazer e, especialmente nos meninos da amostra, enquanto respostas do YSR na amostra de forma geral, indicaram preocupações (como reprovações) e problemas no ambiente escolar (problemas de relacionamento entre estudantes, incluindo referências de violência, além de queixas sobre a jornada escolar integral, falta de motivação e insatisfação com professores). Os meninos da amostra diferentemente das meninas, apresentaram maiores indicativos de estresse com respeito a eventos relacionados à pressão financeira (escala do ASQ em que a metade do subgrupo dos meninos apresentou indicadores de alto índice de estresse).

Os meninos apresentaram tendência maior a problemas psicológicos internalizantes relacionados a transtornos de ansiedade e de transtornos de estresse pós-traumático. Assim, em comparação com as meninas, eles apresentaram-se com mais indicadores problemáticos em termos de desenvolvimento (estresse e problemas internalizantes).

A correlação encontrada no estudo entre dados de estresse (ASQ) e problemas internalizantes (YSR), e entre estresse (ASQ) e total de problemas (YSR), presente no subgrupo dos meninos, mesmo que moderada, pode indicar que a pressão financeira e baixas oportunidades de lazer (indicados como elementos estressores) estejam associadas a problemas psicológicos, o que deve ser mais ampla e profundamente investigado.

A instabilidade emocional no período da adolescência pode ser similar a outros períodos da vida que exigem esforço para enfrentar novas tarefas de desenvolvimento, em princípio estressantes, como a entrada no mundo adulto, a meia idade e a velhice. Por outro lado, a colocação da adolescência como crise (no sentido negativo do termo) que passa com a idade, onde "normal é ser anormal" pode levar a vieses que dificultem o diagnóstico e o tratamento precoce de problemas verdadeiramente psico- 
patológicos ${ }^{29}$. Diante disso, um desafio se impõe enquanto uma refinada tarefa discriminatória: o de não deturpar o entendimento do processo de desenvolvimento positivo da adolescência sob o privilégio de seus aspectos negativos, e nem subdimensionar reais problemas que acometem ado- lescentes, tratando-os como parte inerente e quase natural de uma etapa da vida, vista quase sempre como problemática. Para fazer frente a esse desafio, novas pesquisas devem continuar aprofundando a dimensão de desenvolvimento da vida adolescente.

\section{Referências}

1. Freud A. 0 ego e os mecanismos de defesa. Rio de Janeiro: Civilização Brasileira; 1977

2. Aberastury A, Knobel M. Adolescência normal: um enfoque psicanalítico. Porto Alegre: Artes Médicas; 1981

3. Erikson EH. El ciclo vital completado. México: Paidos Studio; 1990.

4. Erikson EH. Identidade, juventude e crise. $2^{\mathrm{a}}$ ed. Rio de Janeiro: Zahar; 1976.

5. Hoffman L, Paris S, Hall E. Psicología del desarrollo hoy. Madri: McGraw-Hill; 1995.

6. Oliva AD, Gómez AH, Parra AJ, Pertegal MAV, Ríos MB, Antolín LS La promoción del desarrollo adolescente: recursos y estratégias de intervención. Sevilha: Consejería de Salud; 2008.

7. Kimmel D, Weiner I. La adolescencia: una transición del desarrollo. Barcelona: Ariel Psicologia; 1998.

8. Aznar-Farias M, Oliveira-Monteiro NR. Reflexões sobre pró-socialidade, resiliência e psicologia positiva. Rev Bras Ter Cogn. 2006;2(2):39-46.

9. Yunes MA, Miranda AT, Cuello SS. Um olhar ecológico para os riscos e as oportunidades de desenvolvimento de crianças e adolescentes institucionalizados. In: Koller SH, editor. Ecologia do desenvolvimento humano: pesquisa e intervenção no Brasil. São Paulo: Casa do Psicólogo; 2004. p. 197-218.

10. Solano AC. Fundamentos de Psicología Positiva. Buenos Aires: Paidós; 2010.

11. Trianes MV, Muñoz AM, Jimenez M. Competencia social: su educación y tratamiento. Madrid: Pirámide; 1997.

12. Yeates KO, Selman RL. Social Competence in school: towards an integrative developmental model for intervention. Dev Rev. 1989 Mar 9(1):64-100.

13. Rocha MM, Silvares EFM. Competências de jovens brasileiros: fator de proteção para problemas comportamentais. In: Actas do VII Simpósio Nacional de Investigação em Psicologia. Portugal: Universidade do Minho; 2010; $1162-71$

14. Achenbach T, Edelbrock C. The manual for the youth self-report and profile. Burington: University of Vermont; 1987.

15. Poletto M, Koller SH, Dell'Aglio DD. Eventos estressores em crianças e adolescentes em situação de vulnerabilidade social de Porto Alegre. Ciênc Saúde Coletiva. 2009;14(2):455-66.
16. Ferreira HA. Intervenção fisioterapêutica reduz o índice de estresse em vestibulandos. Dissertação (Mestrado) - Universidade Estadual de Campinas, Campinas, 2009.

17. Charmandari E, Tsigos C, Chrousos G. Endocrinology of the stress response. Annu Rev Physiol. 2005;67:259-84.

18. Bronfenbrenner U. A ecologia do desenvolvimento humano: experimentos naturais e planejados. Porto Alegre: Artes Médicas; 2002.

19. Kristensen CH, Leon JS, D'Incao DB, Dell'Aglio DD. Análise da frequência e do impacto de eventos estressores em uma amostra de adolescentes. Interação. 2004;8(1):45-55.

20. Associação Brasileira de Empresas de Pesquisa (ABEP). Critério de Classificação Econômica Brasil. São Paulo; 2012.

21. Byrne DG, Davenport SC, Mazanov J. Profiles of adolescent stress: the development of adolescent stress questionnaire (ASQ). J Adolesc. 2007;30(3):393-416

22. Spadari-Bratfisch, RC, Oliveira-Monteiro NR. Questionário de estresse percebido para adolescentes (ASO). Tradução e adaptação brasileira do "Adolescent Stress Questionnaire" (ASO) de Byrne DG, Davenport SC, Mazanov J. Material não publicado, 2009.

23. Achenbach TM, Rescorla $L A$. Mental Health Practitioners' Guide for the Achenbach System of Empirically Based Assessment (ASEBA). 7th ed. Burlington, VT: University of Vermont, Research Center for Children, Youth \& Families; 2010.

24. Rocha MM, Pereira RF, Arantes MC, Silvares EFM. Guia para profissionais da saúde mental sobre o Sistema Achenbach de Avaliação Empiricamente Baseada (ASEBA). São Paulo. 2010.

25. Rocha MM Evidências de Validade do "Inventário para Adolescentes" (YSR/2001) para a população brasileira. Tese (Doutorado) - Universidade de São Paulo, São Paulo, 2012

26. Assis SG, Avanci JQ, Silva CMFP, Malaquias JV, Santos NC, Oliveira RVC. A representação social do ser adolescente: um passo decisivo na promoção da saúde. Ciênc saúde coletiva. 2003;8(3):669-79.

27. Minayo MCS. Fala galera: juventude, violência e cidadania na cidade do Rio de Janeiro. Brasília: UNESCO; 1999

28. Cavaliere AM. Tempo de escola e qualidade na educação pública. Educ Soc. 2007;28(100):1015-35

29. Aznar-Farias M. Análise de programas de prevenção de problemas comportamentais na adolescência. Tese (Pós-doutorado) - Universidade de Valência, Espanha, 1999. 